\title{
Stereotactic Radiosurgery
}

National Cancer Institute

\section{Source}

National Cancer Institute. Stereotactic Radiosurgery. NCI Thesaurus. Code C15358.

A non-surgical procedure that was developed to deliver one or several maximum dose targeted radiation treatments for cancers in the brain or spine. 